\title{
Thrombin aggravates hypoxia/reoxygenation injury of astrocytes by activating the autophagy pathway mediated by SPRED2
}

\author{
XIAONING WANG ${ }^{1}$, WEIWEI LU ${ }^{1}$, BING LIU ${ }^{1}$ and YUNHE XU ${ }^{2}$ \\ Departments of ${ }^{1}$ Blood Transfusion and ${ }^{2}$ Stomatology, The First Hospital of \\ Jilin University, Changchun, Jilin 130021, P.R. China
}

Received July 28, 2020; Accepted March 24, 2021

DOI: $10.3892 /$ etm.2021.10541

\begin{abstract}
Autophagy plays an important role in ischemia/reperfusion brain injury, however, the signaling pathways involved in cell autophagy are not fully understood. The present study aimed to investigate the roles and molecular mechanisms of thrombin and Sprouty-related EVH1 domain-2 (SPRED2) on autophagy in hypoxia/reoxygenation (H/R) induced astrocytes. Reverse transcription-quantitative PCR and western blot analyses were performed to detect the expression levels of thrombin and SPRED2. Western blot analysis was also performed to detect the protein expression levels of Beclin 1, microtubule-associated protein light chain 3 (LC3)-II and LC3-I. The MTT assay was performed to assess cell viability, while ELISA was performed to determine the supernatant levels of interleukin (IL)-1 $\beta$, IL- 6 and tumor necrosis factor- $\alpha$. The results demonstrated that the effects of $H / R$ induction on inflammatory factor secretion, oxidative stress, autophagy and cell viability in astrocytes were aggravated by thrombin, the effects of which were reversed following SPRED2 knockdown. Taken together, the results of the present study suggest that thrombin aggravates H/R injury in astrocytes by activating the SPRED2-mediated autophagy.
\end{abstract}

\section{Introduction}

Ischemia/reperfusion (I/R) brain injury is one of the leading causes of mortality in cardiovascular and cerebrovascular diseases, with high morbidity and mortality rates worldwide (1). Cerebral ischemic events impact brain physiology (2). An acute ischemic stroke may occur when an artery supplying blood to the brain becomes occluded, leading to excitotoxicity and cell death, ultimately leading to brain tissue death and focal neurological deficits (3).

Correspondence to: Dr Yunhe Xu, Department of Stomatology, The First Hospital of Jilin University, 1 Xinmin Street, Chaoyang, Changchun, Jilin 130021, P.R. China

E-mail: xuyunhe@jlu.edu.cn

Key words: thrombin, hypoxia/reoxygenation, Sprouty-related EVH1 domain-2, autophagy, inflammatory factor, oxidative stress
Prothrombin is cleaved to form thrombin, which acts as a serine protease and belongs to the chymotrypsin family (4). Thrombin activates blood coagulation factors and the anticoagulant protein $\mathrm{C}$ (5). Previous studies have demonstrated that the expression and activity of thrombin increases in tissues and cells following cerebral ischemic injury, and brain cell toxicity is also induced by thrombin $(6,7)$. Bushi et al (7) reported increased thrombin activity in the brain following acute ischemic stroke and demonstrated a close association between thrombin levels and progression of brain damage. Furthermore, thrombin expression is commonly upregulated around the lesion site in intracerebral hemorrhage (8).

Autophagy is a cellular degradation process in which unnecessary or damaged cytoplasmic contents are removed to maintain cellular homeostasis (9). Moderate autophagy has been reported to promote cell survival rate, while excessive autophagy may induce cell cytotoxicity, leading to cell death (10); for example, I/R injury enhanced autophagy (11). Thrombin levels are higher in the ischemic stroke model compared with the control group (12). However, the regulatory association between thrombin and autophagy remains unclear.

Sprouty-related EVH1 domain (SPRED) proteins bind to Ras and Raf-1 and inhibit cytokines, growth factors and the ERK-MAPK pathway (13). A recent study reported that SPRED2 is an essential regulator of cardiac autophagy, whereby its deficiency suppresses autophagy (14). However, the role of SPRED2 in autophagy and the association between SPRED2 and thrombin is yet to be investigated.

'Proper' autophagy is considered to protect against I/R injury, while excessive autophagy contributes to the injury $(15,16)$. For astrocytes, activation of autophagy protects, as well as causes injury (17-19). I/R injury has been reported to activate autophagy in astrocytes, causing astrocyte cell death (17). However, a few studies have demonstrated that the autophagic flux in astrocytes protects brain I/R injury $(18,19)$. Generally, thrombin induces autophagy in astrocytes $(20,21)$. Given that thrombin may contribute to brain I/R injury, the association between thrombin-mediated autophagy in astrocytes and hypoxia/reoxygenation $(\mathrm{H} / \mathrm{R})$ conditions requires investigation.

The present study aimed to investigate the roles and molecular mechanisms of thrombin, SPRED2 and autophagy in $\mathrm{H} / \mathrm{R}$ induced astrocytes and to provide a novel target therapy against cerebral I/R injury. 


\section{Materials and methods}

Cell culture and $H / R$. Rat primary astrocytes were purchased from the American Type Culture Collection and maintained in DMEM (Gibco; Thermo Fisher Scientific, Inc.) supplemented with $10 \%$ fetal bovine serum (FBS; Cytiva), $100 \mathrm{IU} / \mathrm{ml}$ penicillin and $100 \mu \mathrm{g} / \mathrm{ml}$ streptomycin (Sigma-Aldrich; Merck KGaA), at $37^{\circ} \mathrm{C}$ with $5 \% \mathrm{CO}_{2}$ in a humidified atmosphere.

Initially, cells were cultured in DMEM supplemented with $0.5 \%$ FBS for $12 \mathrm{~h}$ and placed in a hypoxia chamber with $5 \% \mathrm{CO}_{2} / 95 \% \mathrm{~N}_{2}$ and incubated at $37^{\circ} \mathrm{C}$ for $24 \mathrm{~h}$. Subsequently, astrocytes were cultured in DMEM supplemented with $10 \% \mathrm{FBS}$ at $37^{\circ} \mathrm{C}$ with $5 \% \mathrm{CO}_{2}$ for $4 \mathrm{~h}$ for reoxygenation.

Cell treatment and transfection. Dabigatran (1 $\mathrm{nM}$; Selleck Chemicals) or thrombin (5 U/ml; Sigma-Aldrich; Merck KGaA) with/without dabigatran were used to treat the induced cells, or the induced cells were treated with thrombin (5 U/ml) with/without 3-MA (Sigma-Aldrich; Merck KGaA).

The H/R induced cells were treated with thrombin (5 U/ml) and transfected with small interfering (si)-SPRED2 or si-negative control (NC; $5 \mathrm{nM}$; all synthesized and purchased from Shanghai GenePharma Co., Ltd.) using Lipofectamine ${ }^{\circledR} 2000$ (Invitrogen; Thermo Fisher Scientific, Inc.), according to the manufacturer's instructions. The Lipofectamine ${ }^{\circledR}$ 2000-si-SPRED2 complex was formed by mixing for $20 \mathrm{~min}$ at room temperature. Then, Lipofectamine $^{\circledR} 2000$-si-SPRED2 complex was added to the cells and the cells were cultured in serum-free Opti-MEM (Gibco; Thermo Fisher Scientific, Inc.) at $37^{\circ} \mathrm{C}$ for $4 \mathrm{~h}$, followed by incubation in serum-containing medium (DMEM; Gibco; Thermo Fisher Scientific, Inc.) at $37^{\circ} \mathrm{C}$ for $72 \mathrm{~h}$. After $48 \mathrm{~h}$, the transfection efficiency was evaluated by reverse transcription-quantitative (RT-qPCR). The sequences were as follows: si-SPRED2, 5'-AUAGCAUUCCAAUACAACCAG-3' and si-NC, 5'-AAUUGACCCAACAAUCACAU-3'.

MTT assay. The MTT assay was performed to assess cell viability. Briefly, astrocytes in different groups were seeded into 96-well plates at a density of $3 \times 10^{3}$ cells/well and were incubated for $48 \mathrm{~h}$ at $37^{\circ} \mathrm{C}$, with $5 \% \mathrm{CO}_{2}$. Subsequently, cells were incubated with $10 \mu \mathrm{l}$ MTT of $5 \mathrm{mg} / \mathrm{ml}$ (Roche Diagnostics) for $4 \mathrm{~h}$ at $37^{\circ} \mathrm{C}$. Following the MTT incubation, the purple formazan crystals were dissolved using $180 \mu \mathrm{l}$ DMSO and viability was subsequently analyzed at a wavelength of $450 \mathrm{~nm}$, using a spectrophotometer (Omega Bio-Tek).

$R T-q P C R$. RT-qPCR analysis was performed to detect the expression levels of SPRED2 and thrombin. Briefly, total RNA was extracted from cells using TRIzol ${ }^{\circledR}$ reagent (Invitrogen; Thermo Fisher Scientific, Inc.). Total RNA was reverse transcribed into cDNA using the PrimeScript RT reagent kit (Applied Biosystems; Thermo Fisher Scientific, Inc.). RT conditions were as follows: $30^{\circ} \mathrm{C}$ for $10 \mathrm{~min}, 42^{\circ} \mathrm{C}$ for $50 \mathrm{~min}$ and $95^{\circ} \mathrm{C}$ for $5 \mathrm{~min}$. PCR reactions were performed in an ABI 7500 Fast RealTime PCR System (Applied Biosystems; Thermo Fisher Scientific, Inc.). qPCR was subsequently performed using the Fast Start Universal SYBR-Green Master (ROX, Takara Biotechnology Co., Ltd.) under the following thermocycling conditions: $95^{\circ} \mathrm{C}$ for $30 \mathrm{sec}, 95^{\circ} \mathrm{C}$ for $5 \mathrm{sec}$ and $60^{\circ} \mathrm{C}$ for $35 \mathrm{sec}$ for 40 cycles, then $72^{\circ} \mathrm{C}$ for $10 \mathrm{~min}$. The following primer sequences were used for qPCR: SPRED2 forward, 5'-TGTGAGCACCGGAAGATTTATACC-3' and reverse, 5'-CGCGGCGGCTTTGTGCTT-3'; thrombin forward, 5'-ATGGCTGCAATCCGAAAGAAG-3' and reverse, 5'-ACAGTAGGGACGTAGACCTCC-3'; and GAPDH forward, 5'-ACCACAGTCCATGCCATCAC-3' and reverse, 5'-TCCACCACCCTGTTGCTGTA-3'. Relative expression levels were calculated using the $2^{-\Delta \Delta C q}$ method and normalized to the internal reference gene GAPDH (22).

Western blotting. Western blot analysis was performed to detect the protein expression levels of SPRED2, microtubule-associated protein light chain 3 (LC3)-II, LC3-I and Beclin 1. Briefly, treated cell lines were harvested and washed with PBS. Total protein was extracted from cells using RIPA buffer (Vazyme Biotech Co., Ltd.). Protein was then quantified using the $\mathrm{BCA}^{\mathrm{TM}}$ Protein Assay kit (Merck KGaA). Samples $(30 \mu \mathrm{g})$ were then separated by 10\% SDS-PAGE (Thermo Fisher Scientific, Inc.). The separated proteins were subsequently transferred onto PVDF membranes (Amersham; Cytiva) and blocked with 5\% non-fat milk at room temperature for $2 \mathrm{~h}$. The membranes were incubated with primary antibodies against thrombin (cat. no. ab92621; 1:1,000), SPRED2 (cat. no. ab153700; 1:500), Beclin 1 (cat. no. ab210498; 1:1,000), LC3-II/I (cat. no. ab62721; 1:2,000) and GAPDH (cat. no. ab181602; 1:10,000) overnight at $4^{\circ} \mathrm{C}$ (all purchased from Abcam). Following the primary incubation, membranes were incubated with a horseradish peroxidase-conjugated secondary antibody (cat. no. ab205718; 1:2,000; Abcam) at $37^{\circ} \mathrm{C}$ for $45 \mathrm{~min}$. Protein bands were visualized by Pierce ECL Western Blotting Substrate (Pierce; Thermo Fisher Scientific, Inc.) using the Bio-Image Analysis System (Bio-Rad Laboratories, Inc.). GAPDH served as the internal control.

ELISA. ELISA kits were used to measure the protein expression levels of interleukin (IL)-1 $\beta$, IL-6 and tumor necrosis factor (TNF) $\alpha$ in the cell supernatant. The following ELISA kits were used: Mouse IL-1 $\beta$ ELISA kit (cat. no. ab197742), Mouse IL-6 ELISA kit (cat. no. ab100712) and Mouse TNF- $\alpha$ ELISA kit (cat. no. ab208348), according to the manufacturer's instructions (all purchased from Abcam).

Measurement of superoxide dismutase (SOD), malondialdehyde (MDA) and reactive oxygen species (ROS) generation. Following H/R treatment, SOD activity was detected using the xanthine oxidase method and MDA content was quantified via the thiobarbituric acid assay (TBA) using MDA (cat. no. A003-1-2) and SOD (cat. no. A001-3-2) kits (Nanjing Jiancheng Bioengineering Institute). ROS generation during reoxygenation was detected using CM-H2DCFDA (cat. no. C6827; Invitrogen; Thermo Fisher Scientific, Inc.).

Statistical analysis. Statistical analysis was performed using SPSS 20.0 software (IBM Corp.). All experiments were repeated in triplicate. Data are presented as the mean \pm standard deviation. Unpaired Student's t-test was used to compare differences between two groups, while one-way ANOVA followed by Tukey's post hoc test were used to compare differences between multiple groups. $\mathrm{P}<0.05$ was considered to indicate a statistically significant difference. 

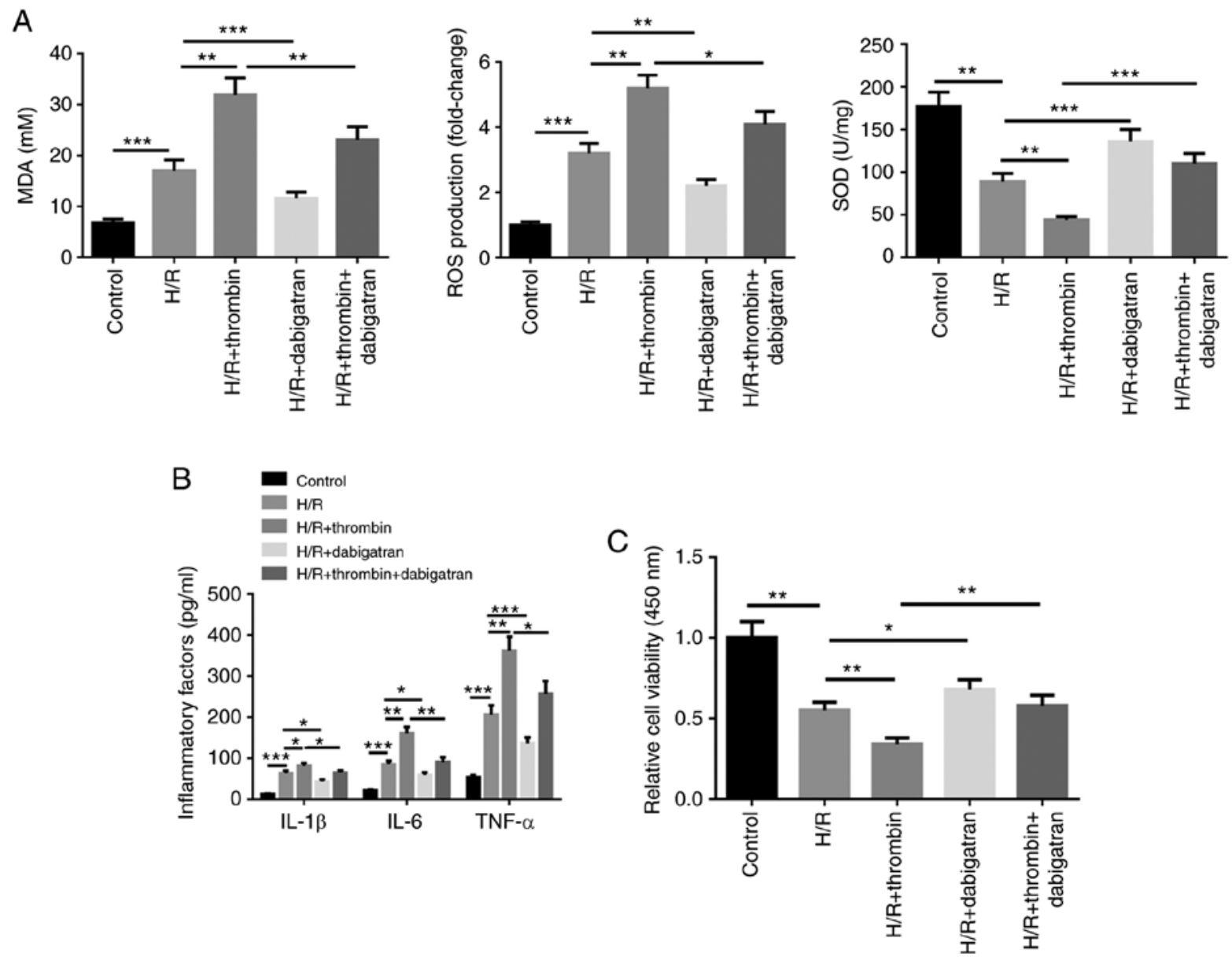

Figure 1. Thrombin aggravates inflammatory factor secretion and oxidative stress in H/R induced astrocytes. (A) The xanthine oxidase method was used to detect SOD activity, the thiobarbituric acid assay was performed to quantify MDA content and CM-H2DCFDA was used to detect ROS generation in H/R induced cells. H/R induced cells were treated with thrombin, dabigatran, and thrombin and dabigatran, respectively. (B) The cell supernatant levels of IL-1 $\beta$, IL-6 and TNF- $\alpha$ were detected via ELISA. (C) The MTT assay was performed to assess cell viability. ${ }^{*} \mathrm{P}<0.05 ;{ }^{* *} \mathrm{P}<0.01 ;{ }^{* * * *} \mathrm{P}<0.001$. H/R, hypoxia/reoxygenation; SOD, superoxide dismutase; MDA, malondialdehyde; ROS, reactive oxygen species; IL, interleukin; TNF, tumor necrosis factor.

\section{Results}

Thrombin aggravates inflammatory factor secretion and oxidative stress in $H / R$ induced astrocytes. The effect of thrombin on astrocytes induced by H/R treatment was assessed by detecting inflammatory factor secretion, oxidative stress and cell viability. The results demonstrated that SOD activity was inhibited following H/R treatment, whereas MDA content and ROS levels increased (Fig. 1A). In addition, the cell supernatant levels of IL-1 $\beta$, IL- 6 and TNF- $\alpha$ increased following H/R treatment (Fig. 1B), whereas cell viability was suppressed, according to the MTT assay (Fig. 1C). In addition, the effect of $H / R$ on astrocytes for SOD activity, MDA content, ROS level, the cell supernatant levels of IL-1 $\beta$, IL- 6 and TNF- $\alpha$, and cell viability was promoted by thrombin, the effects of which were reversed following treatment with dabigatran (Fig. 1A-C). Taken together, these results suggest that thrombin can aggravate inflammatory factor secretion and oxidative stress in H/R induced astrocytes, and inhibit cell viability.

Thrombin aggravates autophagy in $H / R$ induced astrocytes. The effect of thrombin on autophagy in H/R induced astrocytes was investigated. Western blot analysis demonstrated that the protein expression levels of thrombin, SPRED2 and
Beclin 1, and the ratio of LC3-II/I increased following H/R induction, which was promoted by thrombin but attenuated by dabigatran (Fig. 2A). In addition, the regulation of thrombin on the expression levels of SPRED2, Beclin 1 and the ratio of LC3-II/I was reversed by dabigatran. RT-qPCR analysis demonstrated that the mRNA expression levels of thrombin and SPRED2 increased following treatment with thrombin and decreased following treatment with dabigatran, and dabigatran reversed the effects of thrombin on the levels of thrombin and SPRED2 (Fig. 2B). Collectively, these results suggest that thrombin aggravates autophagy in $H / R$ induced astrocytes.

SPRED2 knockdown suppresses the effect of thrombin on inflammatory factor secretion and oxidative stress in $H / R$ induced astrocytes. The molecular mechanism of SPRED2 for thrombin on inflammatory factor secretion, oxidative stress and cell viability in $\mathrm{H} / \mathrm{R}$ induced astrocytes was investigated. As presented in Fig. 3A, SPRED2 knockdown significantly decreased SPRED2 protein expression. MDA content and ROS levels increased following H/R treatment, whereas SOD activity was suppressed (Fig. 3B). In addition, the levels of IL-1 $\beta$, IL- 6 and TNF- $\alpha$ in cell supernatant increased following H/R treatment (Fig. 3C), whereas cell viability was 


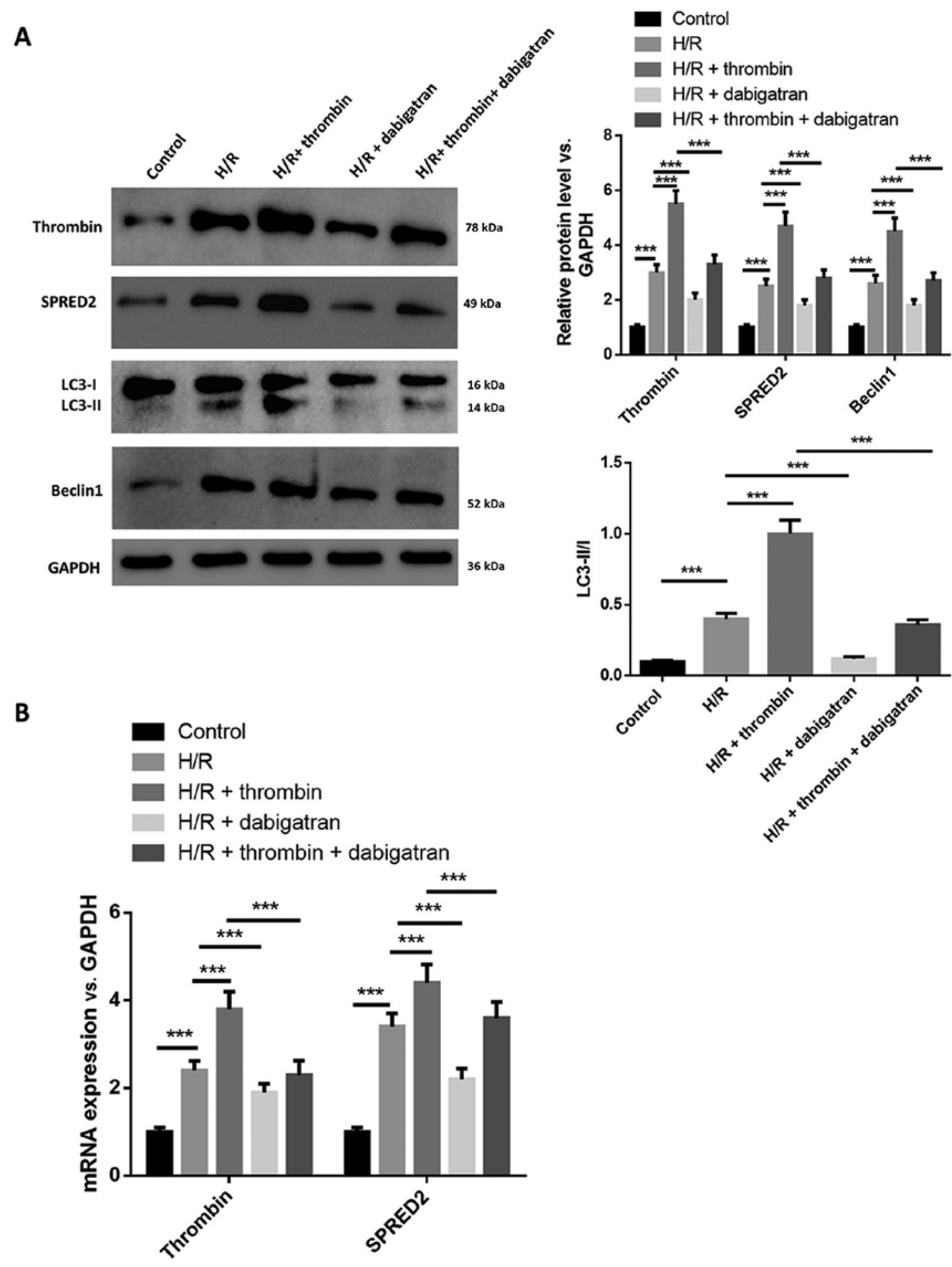

Figure 2. Thrombin aggravates autophagy in H/R induced astrocytes. (A) Western blot analysis was performed to detect the protein expression levels of thrombin, SPRED2, Beclin 1, LC3-II and LC3-I. (B) Reverse transcription quantitative PCR analysis was performed to detect the mRNA expression levels of SPRED2 and thrombin. ${ }^{* * * *} \mathrm{P}<0.001 . \mathrm{H} / \mathrm{R}$, hypoxia/reoxygenation; SPRED2, Sprouty-related EVH1 domain-2; LC3, microtubule-associated protein light chain 3.

inhibited (Fig. 3D). The effect of H/R induction on astrocytes was aggravated following addition of thrombin. However, the expression levels of the inflammatory factors (IL-1 $\beta$, IL-6 and TNF- $\alpha$ ) and oxidative stress markers (MDA and ROS) in cells treated with thrombin were inhibited following SPRED2 knockdown, while the level of SOD was elevated (Fig. 3B and C). Furthermore, the expression levels of the inflammatory factors and oxidative stress markers in cells treated with thrombin were suppressed by autophagy inhibitor
3-MA. Taken together, these results suggest that SPRED2 knockdown suppresses the effect of thrombin on inflammatory factor secretion and oxidative stress in $H / R$ induced astrocytes.

SPRED2 knockdown suppresses the regulation of thrombin on autophagy in $H / R$ induced astrocytes. The regulation of SPRED2 on autophagy in H/R induced astrocytes was investigated. The results demonstrated that thrombin increased 
A
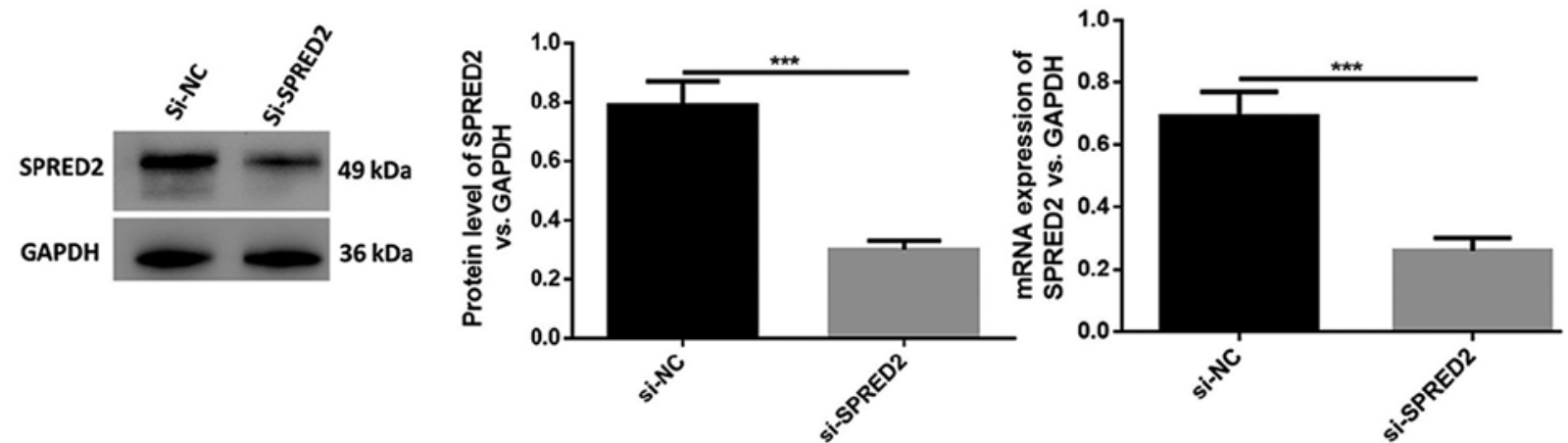

B

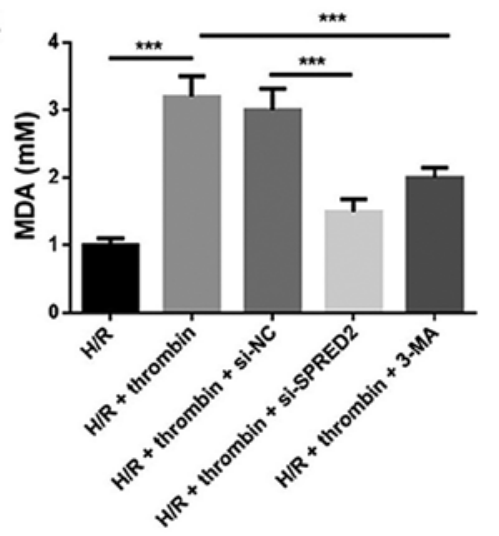

C

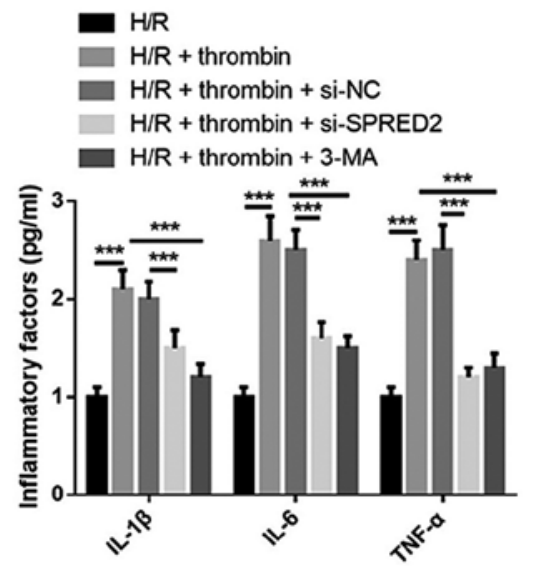

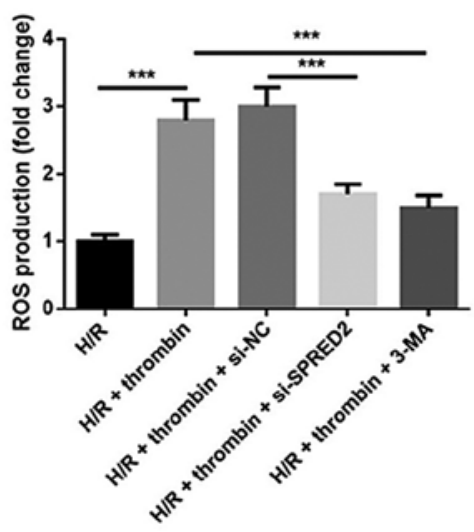

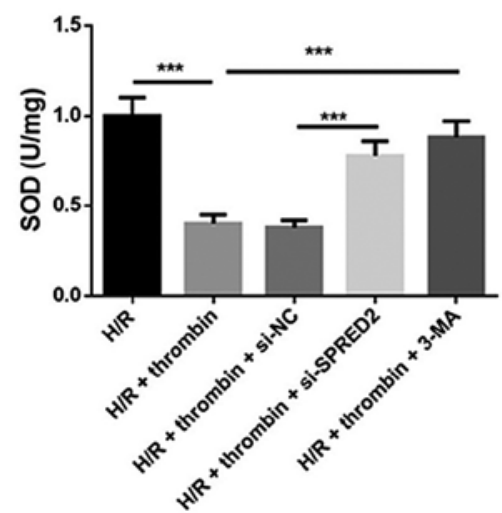

D

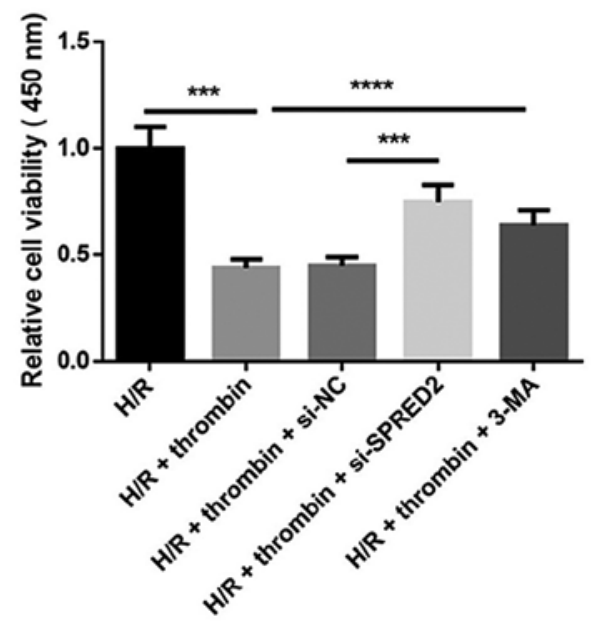

Figure 3. SPRED2 knockdown suppresses the effect of thrombin on inflammatory factor secretion and oxidative stress in H/R induced astrocytes. (A) Western blot and RT-qPCR analysis were performed to determine transfection efficiency of si-SPRED2. (B) The xanthine oxidase method was used to detect SOD activity, the thiobarbituric acid assay was performed to quantify MDA content and CM-H2DCFDA was used to detect ROS generation in H/R induced cells. H/R induced cells were treated with thrombin, and thrombin and 3-MA, and transfected with si-SPRED2 or si-NC. (C) The cell supernatant levels of IL-1 $\beta$, IL-6 and TNF- $\alpha$ were detected via ELISA. (D) The MTT assay was performed to assess cell viability. ${ }^{* * *}$ P $<0.001$. SPRED2, Sprouty-related EVH1 domain-2; H/R, hypoxia/reoxygenation; si, small interfering; SOD, superoxide dismutase; MDA, malondialdehyde; ROS, reactive oxygen species; IL, interleukin; $\mathrm{TNF}$, tumor necrosis factor; $\mathrm{NC}$, negative control.

SPRED2 expression, the ratio of LC3-II/I and Beclin 1 expression (Fig. 4A), the effects of which were reversed following transfection with si-SPRED2. The effect of thrombin on cells induced by $\mathrm{H} / \mathrm{R}$ was suppressed following treatment with 3-MA. RT-qPCR analysis demonstrated that SPRED2 expression decreased following transfection with si-SPRED2, and 3-MA reversed the effect of thrombin on cells with $\mathrm{H} / \mathrm{R}$ induction (Fig. 4B). Collectively, these results suggest that thrombin regulation on autophagy in $H / R$ induced astrocytes can be inhibited by silencing SPRED2.

\section{Discussion}

Irreversible damages, such as oxidative stress, death associated with inflammation, neuronal injury and excitotoxicity, are the results of I/R brain injury (23). Oxidative stress plays an important role in the development of the ischemic cascade. Thus, the intervention of oxidative damage may prevent diseases induced by oxidative stress (24). Furthermore, inflammation plays an important role in the pathophysiology of cerebral ischemia, as well as ischemic cerebrovascular diseases (25). The roles and 
A

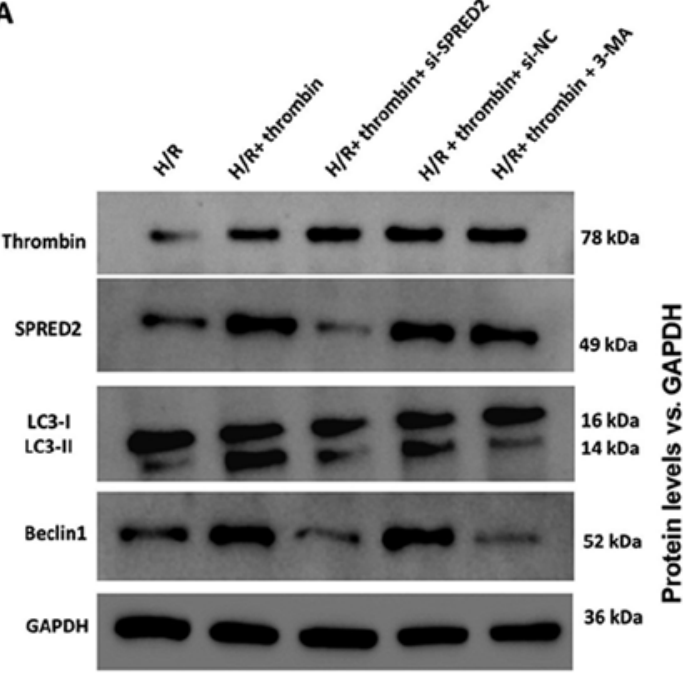

ש $H / R$

- $H / R+$ thrombin

H/R + thrombin + si-SPRED2

- $H / R+$ thrombin + si-NC

- H/R + thrombin + 3-MA
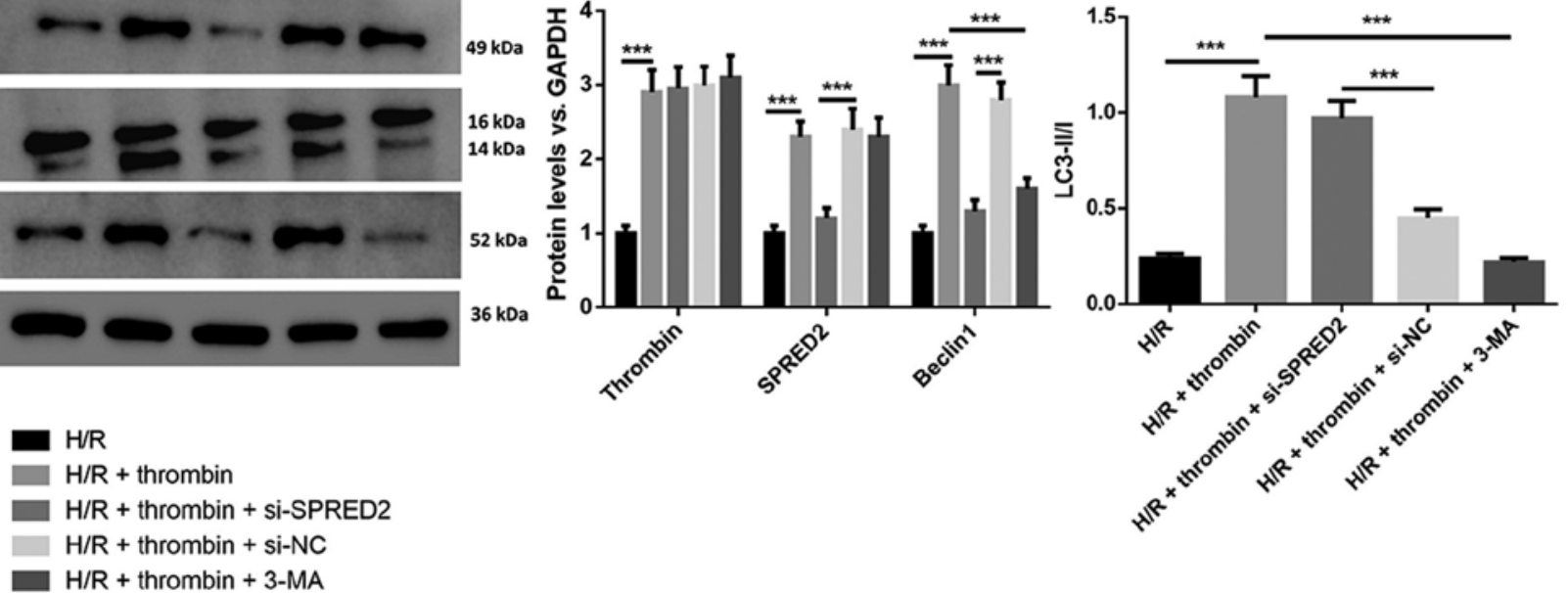



$H / R+$ thrombin + 3-MA

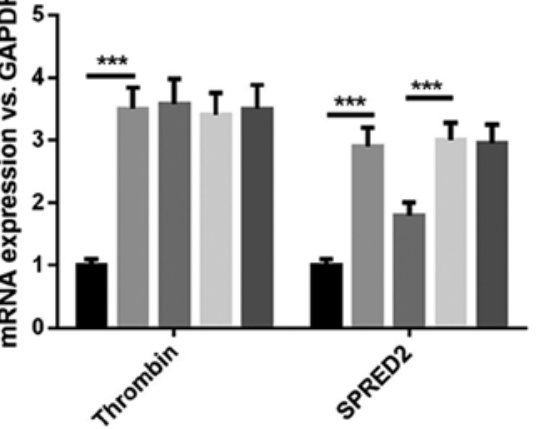

Figure 4. Regulation of thrombin on autophagy in H/R induced astrocytes is suppressed by silencing SPRED2. (A) Western blot analysis was performed to detect the protein expression levels of thrombin, SPRED2, Beclin 1, LC3-II and LC-3I. (B) Reverse transcription-quantitative PCR analysis was performed to detect the mRNA expression levels of SPRED2 and thrombin. ${ }^{* * *} \mathrm{P}<0.001$. H/R, hypoxia/reoxygenation; SPRED2, Sprouty-related EVH1 domain-2; LC3, microtubule-associated protein light chain 3; si, small interfering; NC, negative control.

molecular mechanisms of thrombin and SPRED2 in the I/R induced rat astrocytes model were investigated in the present stud.

Increasing evidence suggest that thrombin is a mediator for cerebrovascular inflammation and hypoxia, with obvious value in hypoxia, inflammation and oxidative stress $(26,27)$. A previous study reported that thrombin promotes macrophage differentiation into an M1-like phenotype, closely associated with the expression of classical pro-inflammatory markers (28). Another study demonstrated that thrombin promotes inflammatory gene expression and enhances sustained signaling in astrocytes (29). The results of the present study demonstrated that thrombin aggravates inflammatory factor secretion and oxidative stress induced by $\mathrm{H} / \mathrm{R}$ in astrocytes, which is consistent with the aforementioned reports $(28,29)$. Autophagy usually occurs in response to cellular stresses, including oxidative stress, infection and nutrient starvation $(15,30)$. However, the molecular mechanism of autophagy remains unknown. Hu et al (21) reported that thrombin increases the ratio of LC3-II/LC3-I and the level of cathepsin D; thus, thrombin activates autophagy and plays an important role in
ICH-induced autophagy. Another report demonstrated that the level of perihematomal neuron autophagy was correlated with thrombin-antithrombin plasma levels in patients with intracerebral hemorrhage (31). The present study demonstrated that thrombin aggravated autophagy in $\mathrm{H} / \mathrm{R}$ induced astrocytes.

SPRED2 belongs to a family of proteins containing a cysteine-rich domain and is widely expressed in several tissues, including the brain (32). SPRED2 regulates adipose tissue inflammation and metabolic abnormalities induced by a high-fat diet in mice (33). Downregulated SPRED2 expression attenuates epithelial cell injury and inflammation in dextran sulfate sodium-induced acute colitis in mice (34). Itakura et al (35) reported that inflammatory responses decrease by inhibiting SPRED2 from protecting mice from polymicrobial sepsis by activating the ERK/MAPK pathway. The results of the present study demonstrated that the promotion of inflammatory factor secretion and oxidative stress in $\mathrm{H} / \mathrm{R}$ induced astrocytes by thrombin was suppressed following SPRED2 knockdown. However, very few studies have investigated the role of SPRED2 in autophagy (32-35). In addition, autophagy may be a promising target for promoting 
SPRED2-mediated antitumor activity (36). The results of the present study demonstrated that SPRED2 knockdown suppressed the promotional effect of thrombin on autophagy in $\mathrm{H} / \mathrm{R}$ induced astrocytes.

The present study is not without limitations. First, deeper insights on the regulatory association between SPRED2 and autophagy in the I/R process were not investigated in the present study. Secondly, it is unclear whether other signaling pathways are involved in thrombin aggravated I/R injury. Thus, further studies are required to confirm the results presented here.

In conclusion, the results of the present study demonstrated that inflammatory factor secretion, oxidative stress and autophagy in $\mathrm{H} / \mathrm{R}$ induced astrocytes were aggravated by thrombin, which was inhibited by SPRED2 knockdown. To the best of our knowledge, the present study is the first to demonstrate H/R injury of astrocytes aggravated by thrombin by activating the autophagy pathway mediated by SPRED2. This research will help to identify novel targets against I/R brain injury.

\section{Acknowledgements}

Not applicable.

\section{Funding}

The present study was funded by the Achievement Transformation Project of the First Hospital of Jilin University (grant no. JDYYZH-2102057).

\section{Availability of data and materials}

The datasets used and/or analyzed during the current study are available from the corresponding author on reasonable request.

\section{Authors' contributions}

XW designed and performed the experiments, analyzed data and wrote the manuscript. WL and BL performed experiments and collected and analyzed data. YX performed data analysis and reviewed and revised the manuscript. WL and YX confirm the authenticity of all the raw data. All authors read and approved the final manuscript.

\section{Ethics approval and consent to participate}

Not applicable.

\section{Patient consent for publication}

Not applicable.

\section{Competing interests}

The authors declare that they have no competing interests.

\section{References}

1. Disdier C, Chen X, Kim JE, Threlkeld SW and Stonestreet BS: Anti-cytokine therapy to attenuate ischemic-reperfusion associated brain injury in the perinatal period. Br Sci 8: 101, 2018.
2. de la Monte SM, Gallucci GM, Lin A, Tong M and Stonestreet BS: Critical shifts in cerebral white matter lipid profiles after ischemic-reperfusion brain injury in fetal sheep as demonstrated by the positive ion mode MALDI-mass spectrometry. Cell Medicine: Feb 7, 2020 (Epub ahead of print).

3. Vermani B, Mukherjee S, Kumar G and Patnaik R: Prolactin attenuates global cerebral ischemic injury in rat model by conferring neuroprotection. Brain Inj 34: 685-693, 2020

4. Scalambrino E, Padovan L, Chantarangkul V, Clerici M, Artoni A, Peyvandi F and Tripodi A: Responsiveness of the activated partial thromboplastin time and dilute thrombin time to argatroban: Results of an in vitro study. Int J Lab Hematol 42: e128-e131, 2020

5. Zavyalova EG, Ustinov NB and Kopylov AM: Exploring the efficiency of thrombin inhibitors with a quantitative model of the coagulation cascade. FEBS Lett 594: 995-1004, 2020.

6. Bushi D, Chapman J, Wohl A, Stein ES, Feingold E and Tanne D: Apixaban decreases brain thrombin activity in a male mouse model of acute ischemic stroke. J Neurosci Res 96: 1406-1411, 2018.

7. Bushi D, Stein ES, Golderman V, Feingold E, Gera O, Chapman J and Tanne D: A linear temporal increase in thrombin activity and loss of its receptor in mouse brain following ischemic stroke. Front Neurol 8: 138, 2017.

8. $\mathrm{Hu} \mathrm{S}$, Wu G, Zheng J, Liu X and Zhang Y: Astrocytic thrombin-evoked VEGF release is dependent on p44/42 MAPKs and PAR1. Biochem Biophys Res Commun 509: 585-589, 2019.

9. Rao JY, Wang Q, Wang YC, Xiang F, Tian XC, Liu DH and Dong Z: $\beta$-caryophyllene alleviates cerebral ischemia/reperfusion injury in mice by activating autophagy. Zhongguo Zhong Yao Za Zhi 45: 932-936, 2020 (In Chinese).

10. Zhang Y, Tian Z, Wan H, Liu W and Ma G: Deltonin ameliorates cerebral ischemia/reperfusion injury in correlation with modulation of autophagy and inflammation. Neuropsychiatr Dis Treat 16: 871-879, 2020 .

11. Qiu R, Li W and Liu Y: MicroRNA-204 protects H9C2 cells against hypoxia/reoxygenation-induced injury through regulating SIRT1-mediated autophagy. Biomed Pharmacother 100: 15-19, 2018.

12. Bushi D, Chapman J, Katzav A, Shavit-Stein E, Molshatzki N, Maggio N and Tanne D: Quantitative detection of thrombin activity in an ischemic stroke model. J Mol Neurosci 51: 844-850, 2013.

13. Susanto A, Zhao G, Wazin F, Feng Y, Rasko JEJ, Bailey CG and Lovicu FJ: Spred negatively regulates lens growth by modulating epithelial cell proliferation and fiber differentiation. Exp Eye Res 178: 160-175, 2019.

14. Ullrich M, Aßmus B, Augustin AM, Häbich H, Abeßer M, Machado JM, Werner F, Erkens R, Arias-Loza AP, Umbenhauer S, et al: SPRED2 deficiency elicits cardiac arrhythmias and premature death via impaired autophagy. J Mol Cell Cardiol 129: 13-26, 2019.

15. Bu Q, Liu X, Zhu Y, Liu Y and Wang Y: w007B protects brain against ischemia-reperfusion injury in rats through inhibiting inflammation, apoptosis and autophagy. Brain Res 1558: 100-108, 2014.

16. Zhang X, Yan H, Yuan Y, Gao J, Shen Z, Cheng Y, Shen $\mathrm{Y}$, Wang RR, Wang $\mathrm{X}, \mathrm{Hu} \mathrm{WW}$, et al: Cerebral ischemia-reperfusion-induced autophagy protects against neuronal injury by mitochondrial clearance. Autophagy 9: 1321-1333, 2013.

17. Pan R, Timmins GS, Liu W and Liu KJ: Autophagy mediates astrocyte death during zinc-potentiated ischemia-reperfusion injury. Biol Trace Elem Res 166: 89-95, 2015.

18. Liu X, Tian F, Wang S, Wang F and Xiong L: Astrocyte autophagy flux protects neurons against oxygen-glucose deprivation and ischemic/reperfusion injury. Rejuvenation Res 21: 405-415, 2018.

19. Zhu C, Zhou Q, Luo C and Chen Y: Dexmedetomidine protects against oxygen-glucose deprivation-induced injury through inducing astrocytes autophagy via TSC2/mTOR pathway. Neuromolecular Med 22: 210-217, 2020.

20. Hu S, Wu G, Ding X and Zhang Y: Thrombin preferentially induces autophagy in glia cells in the rat central nervous system. Neurosci Lett 630: 53-58, 2016.

21. Hu S, Xi G, Jin H, He Y, Keep RF and Hua Y: Thrombin-induced autophagy: A potential role in intracerebral hemorrhage. Brain Res 1424: 60-66, 2011. 
22. Livak KJ and Schmittgen TD: Analysis of relative gene expression data using real-time quantitative PCR and the 2(-Delta Delta C(T)) method. Methods 25: 402-408, 2001.

23. Li Q, Ye T, Long $\mathrm{T}$ and Peng X: Ginkgetin exerts antiinflammatory effects on cerebral ischemia/reperfusion-induced injury in a rat model via the TLR4/NF- $\kappa \mathrm{B}$ signaling pathway. Biosci Biotechnol Biochem 83: 675-683, 2019.

24. Kar F, Hacioglu C, Senturk H, Donmez DB and Kanbak G: The role of oxidative stress, renal inflammation, and apoptosis in post ischemic reperfusion injury of kidney tissue: The protective effect of dose-dependent boric acid administration. Biol Trace Elem Res 195: 150-158, 2020.

25. Wang YS, Li YX, Zhao P, Wang HB, Zhou R, Hao YJ, Wang J, Wang SJ, Du J, Ma L, et al: Anti-inflammation effects of oxysophoridine on cerebral ischemia-reperfusion injury in mice. Inflammation 38: 2259-2268, 2015.

26. Tripathy D, Sanchez A, Yin X, Luo J, Martinez J and Grammas P: Thrombin, a mediator of cerebrovascular inflammation in AD and hypoxia. Front Aging Neurosci 5: 19, 2013.

27. Ohshiro K, Bui-Nguyen TM, Divijendra Natha RS, Schwartz AM, Levine P and Kumar R: Thrombin stimulation of inflammatory breast cancer cells leads to aggressiveness via the EGFR-PAR1-Pak1 pathway. Int J Biol Markers 27: e305-e313, 2012.

28. López-Zambrano M, Rodriguez-Montesinos J, Crespo-Avilan GE Muñoz-Vega M and Preissner KT: Thrombin promotes macrophage polarization into M1-like phenotype to induce inflammatory responses. Thromb Haemost 120: 658-670, 2020.

29. Dusaban SS, Kunkel MT, Smrcka AV and Brown JH: Thrombin promotes sustained signaling and inflammatory gene expression through the CDC 25 and Ras-associating domains of phospholipase C $\epsilon$. J Biol Chem 290: 26776-26783, 2015.

30. Yang Y, Karsli-Uzunbas G, Poillet-Perez L, Sawant A, Hu ZS, Zhao Y, Moore D, Hu W, White E and White E: Autophagy promotes mammalian survival by suppressing oxidative stress and p53. Genes Dev 34: 688-700, 2020.
31. Wu C, Yan X, Liao Y, Liao L, Huang S, Zuo Q, Zhou L, Gao L, Wang Y, Lin J, Li S, Wang K, Ge X, Song H, Yang R and $\mathrm{Lu}, \mathrm{F}$ : Increased perihematomal neuron autophagy and plasma thrombin-antithrombin levels in patients with intracerebral hemorrhage: An observational study. Medicine 98: (39) 17130, 2019.

32. Kawazoe T and Taniguchi K: The Sprouty/Spred family as tumor suppressors: Coming of age. Cancer Sci 110: 1525-1535, 2019.

33. Ohkura T, Yoshimura T, Fujisawa M, Ohara T, Marutani R, Usami K and Matsukawa A: Spred2 regulates high fat diet-induced adipose tissue inflammation, and metabolic abnormalities in mice. Front Immunol 10: 17, 2019.

34. Takahashi S, Yoshimura T, Ohkura T, Fujisawa M, Fushimi S, Ito T,Itakura J,Hiraoka S, Okada H, Yamamoto Kand Matsukawa A: A novel role of Spred2 in the colonic epithelial cell homeostasis and inflammation. Sci Rep 6: 37531, 2016.

35. Itakura J, Sato M, Ito T, Mino M, Fushimi S, Takahashi S, Yoshimura T and Matsukawa A: Spred2-deficiecy protects mice from polymicrobial septic peritonitis by enhancing inflammation and bacterial clearance. Sci Rep 7: 12833, 2017.

36. Jiang K, Liu M, Lin G, Mao B, Cheng W, Liu H, Gal J, Zhu H, Yuan Z, Deng W, et al: Tumor suppressor Spred2 interaction with LC3 promotes autophagosome maturation and induces autophagy-dependent cell death. Oncotarget 7: 25652-25667, 2016.

(i) $($ This work is licensed under a Creative Commons Attribution-NonCommercial-NoDerivatives 4.0 International (CC BY-NC-ND 4.0) License. 